\title{
Influence du taux d'acide alpha-linolénique de l'aliment sur la teneur en oméga-3 et les caractéristiques hédoniques de la viande de lapin. Revue bibliographique
}

\author{
Benoît TEILLET ${ }^{1}$ \\ Michel COLIN ${ }^{1}$ \\ Anne Yvonne PRIGENT ${ }^{2}$ \\ 1 COPRI, \\ Coat Izella, \\ 29830 Ploudalmézeau, \\ France 3 \\ <copri@wanadoo.fr> \\ ${ }^{2}$ EARL 3L, \\ Coat Izella, \\ 29830 Ploudalmézeau (France) \\ Article reçu le $1^{\mathrm{er}}$ mars 2011 \\ Accepté le 5 septembre 2012
}

\begin{abstract}
Influence of the feed linolenic acid level on the Omega 3 contend and the hedonic characteristics of the rabbit meat. A review

Several publications are dealing with the influence of the omega 3 level in the feed, more particularly the alpha-linolenic acid level, on the rabbit meat level in this fatty acid, but only few of them deal with the use of the flaxseed. This review is a synthesis of the effects of the level of alpha-linolenic acid brought by the flaxseed on the omega 3 level of the rabbit meat. It shows a direct connection between the alpha-linolenic acid level in the feed and the omega 3 content of the rabbit meat. Besides, this enrichment decreases the saturated fatty acids level, the omega 6 level and consequently the omega 6/omega 3 ratio below 4 . In consequence, the contribution of around $0.65 \%$ of alpha-linolenic acid in the feed by incorporation of flaxseed in the feed allows to bring in average $967 \mathrm{mg}$ of omega 3 for $100 \mathrm{~g}$ of back or $459 \mathrm{mg}$ for $100 \mathrm{kcal}$ and $1004 \mathrm{mg}$ of omega 3 for $100 \mathrm{~g}$ of shoulder or $483 \mathrm{mg}$ for $100 \mathrm{kcal}$ and consequently to claim the allegation "rich in omega 3 ". This enrichment doesn't modify the hedonic characteristics of the rabbit meat.
\end{abstract}

Key words: meat quality, rabbits, lipids, omega 3, alpha-linolenic acid, extruded flax

linolénique (notés "ALA » dans le reste du texte) de l'aliment sur les qualités hédoniques de la viande de lapin ont été peu étudiés (Dal Bosco et al., 2003).

Nous avons donc cherché à déterminer dans quelle mesure un accroissement du taux d'ALA de I'alimentation des lapins obtenu par addition de graines de lin est susceptible d'accroître le taux d'oméga-3 du râble, de l'épaule et de la cuisse. Une fois cette corrélation démontrée, l'impact d'un enrichissement en ALA de I'aliment sur la composition globale des viandes et leurs caractéristiques hédoniques a également été étudié.

\section{Matériel et méthodes}

Cette synthèse bibliographique se déroule en 3 parties. La première partie consiste à mettre en évidence la corrélation entre le taux d'oméga-3 dans I'aliment via le taux d'ALA, puisque la source d'oméga-3 est une source végétale terrestre, et le taux d'oméga3 dans la viande de lapin, puisque $98 \%$ des oméga- 3 contenus dans la viande sont de I'ALA (Colin et al., 2012; Combes et Cauquil, 2006). Pour cela, nous utilisons 3 sources bibliographiques: Dal Bosco et al. (2003), Colin et al. (2005) et Colin et al. (2010). Ensuite, les conséquences d'un enrichissement de l'aliment en oméga-3 sur la composition globale de la viande de lapin ont été abordées par I'utilisation d'une banque de données réalisée à I'EARL 3L et publiée partiellement dans le travail de Colin et al. (2010). Enfin, les effets de cet enrichissement sur les qualités hédoniques ont été étudiés sur deux des essais utilisés en première partie.

Pour citer cet article : Teillet B, Colin M, Prigent AY. Influence du taux d'acide alpha-linolénique de l'aliment sur la teneur en oméga-3 et les caractéristiques hédoniques de la viande de lapin. Revue bibliographique. OCL $2013 ; 20(1): 55-60$. doi : 10.1684/ocl.2012.0480 


\section{Animaux}

Dans les essais de l'EARL 3L, 1315 lapins de 38 jours de souche Hyplus ont été répartis entre différents groupes (de 2 à 5). Chaque groupe de lapins a reçu un aliment se différenciant par sa teneur en ALA. Ils ont été abattus à l'âge de 75 jours dans un abattoir commercial et des carcasses ont été prélevées de façon aléatoire dans chaque régime. Elles ont été envoyées au laboratoire CARSO à Quimper (France) pour analyses chimiques en pool et tests hédoniques (tableau 1).

Dans l'essai de Dal Bosco et al. (2003), 120 lapins de souche Martini ont été répartis au sevrage entre 2 groupes. Chaque groupe de lapins reçoit un aliment se différenciant par sa teneur en ALA. Ils ont été abattus à l'âge de 85 jours et 20 carcasses par groupe ont été utilisées pour analyses chimiques (tableau 1).

\section{Aliments}

La source d'ALA utilisée pour augmenter le niveau de cet acide gras dans les aliments est uniquement la graine de lin, crue dans l'essai de Dal Bosco et al.
(2003), extrudée (Tradi-lin) dans les travaux de I'EARL 3L. Le taux d'incorporation de cette matière première $a$ volontairement été ajusté pour faire varier le taux d'ALA de I'aliment. Afin d'approcher dans les meilleures conditions les effets d'une variation de l'apport en ALA des aliments sur les caractéristiques de la viande de Lapin, le régime témoin des essais 4 et 5 a volontairement été formulé à un taux faible d'ALA (tableau 2). Tous les essais sont réalisés sans luzerne à I'EARL 3L (Colin et al., 2005 ) et avec $35 \%$ de luzerne dans l'essai de Dal Bosco et al. (2003).

\section{Analyses chimiques et hédoniques}

Selon les essais, des épaules, des cuisses et des râbles ont été prélevés pour chaque lot. Chacun des 3 types de morceaux a fait l'objet d'une analyse chimique réalisée au laboratoire CARSO à Quimper (France) sur échantillon moyen portant sur :

- le taux d'humidité selon la norme ISO 1442:1997 de février 1997 ;

- le taux de matières grasses libres selon la norme ISO 1444:1996 d'avril 1996 ;
- le taux de protéines selon la norme ISO 937 :1978 de décembre 1978 ;

- une caractérisation du profil d'acide gras réalisée selon la norme NF EN ISO 5508-5509.

Les tests hédoniques ont été réalisés dans les essais 4 et 5 sous forme d'une épreuve de dégustation réalisée auprès de 150 consommateurs ( 60 par essai et par morceau) de plus de 18 ans, moitié femmes et moitié hommes et consommateurs de viande de lapin. Les morceaux ont été cuits à $250^{\circ} \mathrm{C}$ dans des fours vapeur faible flux en étant recouverts d'une feuille d'aluminium.

Pour chaque partie de lapin, tous les consommateurs ont reçu un morceau correspondantaux 2 traitements. Chaque morceau a fait l'objet d'une notation de 1 (Extrêmement désagréable) à 7 (Extrêmement agréable) pour 5 critères: appréciation globale, aspect visuel, odeur, qualité du goût, texture. Des questions ouvertes permettaient aux juges d'expliquer leur choix si nécessaire.

\section{Analyses statistiques}

Les résultats des tests hédoniques ont été analysés selon un test de Student.

Tableau 1. Résultats des tests hédoniques.

\begin{tabular}{|llllll|}
\hline Références & Année & Génétique & $\begin{array}{l}\text { Nombre } \\
\text { de lapins }\end{array}$ & $\begin{array}{l}\text { Nombre de lapins } \\
\text { analysés }\end{array}$ & $\begin{array}{l}\text { Réalisation d'un } \\
\text { test hédonique }\end{array}$ \\
\hline Dal Bosco et al., 2003 & 2003 & Martini - Italie & 120 & 80 & Non \\
\hline EARL 3L- Essai $n^{\circ}$ 1/Colin et al., 2010 & 2003 & Hyplus - France & 240 & 20 & Non \\
\hline EARL 3L- Essai $n^{\circ}$ 2/Colin et al., 2010 & 2003 & Hyplus - France & 86 & 20 & Non \\
\hline EARL 3L- Essai $n^{\circ}$ 3/Colin et al., 2010 & 2004 & Hyplus - France & 76 & 20 & Non \\
\hline EARL 3L- Essai $n^{\circ}$ 4/Colin et al., 2005 & 2005 & Hyplus - France & 400 & 70 & Oui \\
\hline EARL 3L- Essai $n^{\circ}$ 5/Colin et al., 2010 & 2007 & Hyplus - France & 513 & 20 & Oui \\
\hline TOTAL & & & 1435 & 230 & 2 \\
\hline
\end{tabular}

Tableau 2. Description des essais.

\begin{tabular}{|llll|}
\hline \multirow{2}{*}{ Références } & \multirow{2}{*}{ Source d'ALA } & \multicolumn{2}{l|}{ Taux d'ALA dans I'aliment } \\
\cline { 3 - 4 } & Standard & Graine de lin \\
\hline Dal Bosco et al., 2003 & Graine de lin & $0,41 \%$ & $1,11 \%$ \\
\hline EARL 3L- Essai $n^{\circ}$ 1/Colin et al., 2010 & Graine de lin extrudé & $0,22 \%$ & $0,81 \% / 0,81 \% / 1,27 \% / 1,36 \%$ \\
\hline EARL 3L- Essai $n^{\circ}$ 2/Colin et al., 2010 & Graine de lin extrudé & $0,22 \%$ & $0,71 \%$ \\
\hline EARL 3L- Essai $n^{\circ}$ 3/Colin et al., 2010 & Graine de lin extrudé & $0,23 \%$ & $0,87 \%$ \\
\hline EARL 3L- Essai $n^{\circ}$ 4/Colin et al., 2005 & Graine de lin extrudé & $0,06 \%$ & $0,80 \%$ \\
\hline EARL 3L- Essai $n^{\circ}$ 5/Colin et al., 2010 & Graine de lin extrudé & $0,03 \%$ & $0,65 \% / 0,66 \%$ \\
\hline
\end{tabular}




\section{Résultats et discussion}

Corrélation entre le taux d'ALA de I'aliment et celui de la viande

La compilation des différentes données est présentée sous forme de graphique selon la méthode publiée par Combes et Dalle Zotte (2005). Elle montre ainsi une corrélation importante entre le taux d'ALA de l'aliment et le taux d'oméga-3 de la viande de lapin, quelque soit le morceau : $\mathrm{R}^{2}=0,79$ pour le râble, $\mathrm{R}^{2}=0,81$ pour l'épaule et $\mathrm{R}^{2}=0,72$ pour la cuisse (figures 1, 2 et 3). L'enrichissement de I'aliment en ALA modifie la composition des lipides de la viande de lapin par un accroissement du taux d'oméga-3.

\section{Conséquence sur la composition globale de la viande de lapin}

La seconde partie de ce travail a consisté à comparer la composition globale entre un lapin standard (" std "), c'està-dire nourri avec un aliment dont le taux d'oméga-3 est inférieur à 0,2 \%, à un lapin appelé "Lin », nourri avec un aliment apportant entre 0,5\% et 0,8\% d'oméga-3. Pour cela, l'ensemble des analyses réalisées à I'EARL $3 \mathrm{~L}$ depuis 2003, soit 62 analyses sur 686 morceaux, ont été utilisées. Le nombre d'échantillon concernant les lapins standards est beaucoup plus faible que celui des lapins recevant un aliment avec lin, mais les valeurs moyennes et les écartstypes sont conformes à ce que l'on peut trouver dans la littérature (Combes et Dalle Zotte, 2005; Gigaud et Combes, 2008 ; Mourot, 2010), ce qui légitime les comparaisons ainsi réalisées (tableau 3).

L'enrichissement de I'aliment en ALA entraîne une hausse des lipides dans le râble et l'épaule puisque l'aliment est plus riche en lipides. Par contre, en accord avec les données de Bernardini et al. (1997), le phénomène est inversé pour la cuisse. De plus, cet enrichissement en ALA diminue systématiquement le taux d'acides gras saturés et augmente le taux d'acides gras insaturés, observations confirmées par Bernardini et al (1999) et Dal Bosco et al (2003). II décroît aussi systématiquement le taux d'oméga 6. Ainsi le rapport oméga 6/oméga-3 se situe en dessous de 4 (tableau 4).

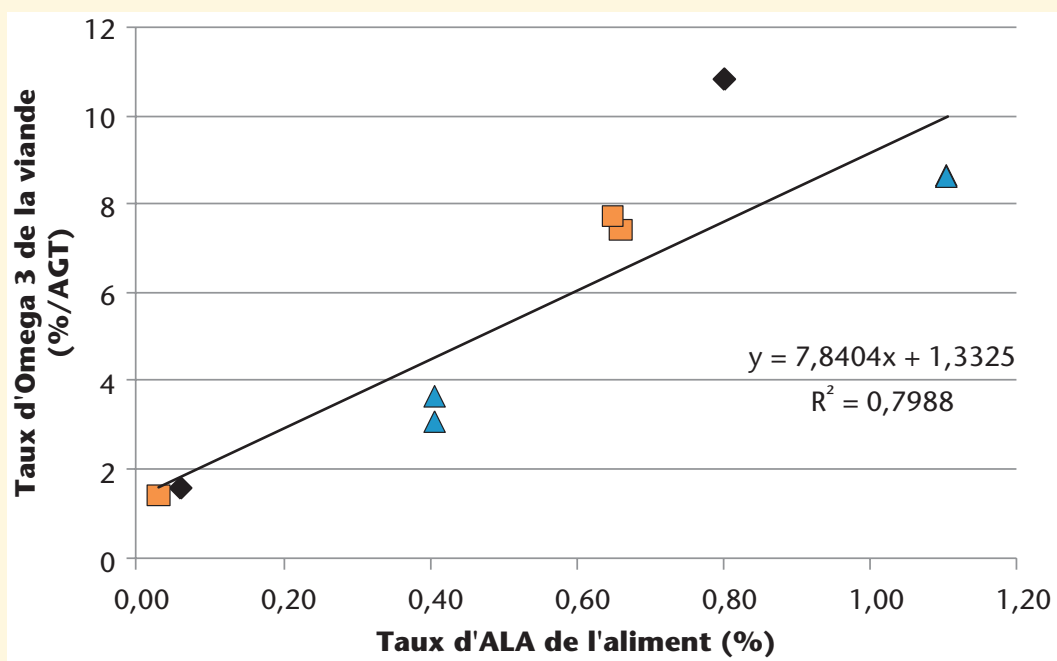

$\begin{array}{ll}\triangle \text { Dal Bosco et al., } 2003 & \triangle \text { EARL 3L - Essai n¹ - Colin et al., } 2010 \\ \text { EARL 3L - Essai n² - Colin et al., 2010 } & \square \text { EARL 3L - Essai n³ - Colin et al., } 2010 \\ - \text { EARL 3L - Essai } n^{\circ} 4 \text { - Colin et al., 2005 } & \square \text { EARL 3L - Essai } n^{\circ} 5 \text { - Colin et al., } 2010\end{array}$

Figure 1. Relation entre le taux d'ALA de I'aliment et la teneur en ALA des graisses du râble.

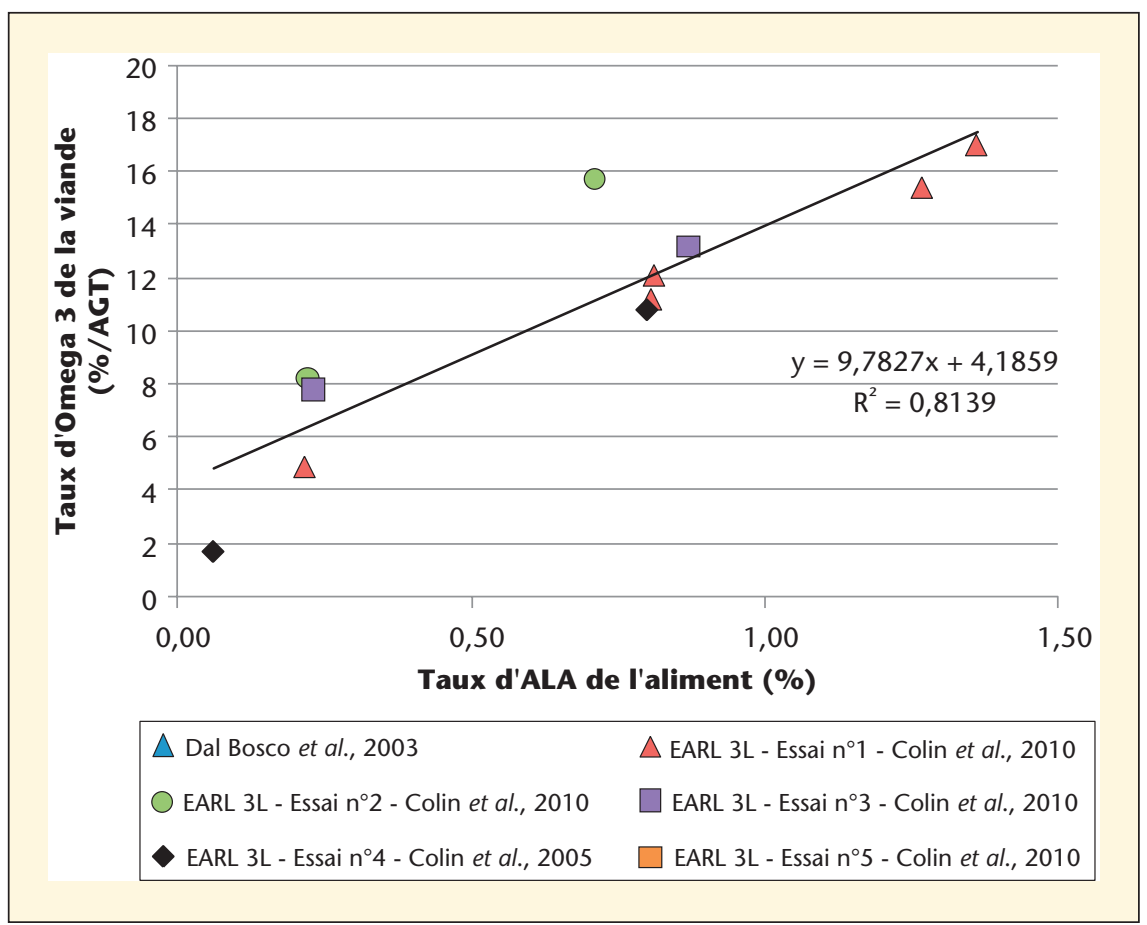

Figure 2. Relation entre le taux d'ALA de l'aliment et la teneur en ALA des graisses de l'épaule. 


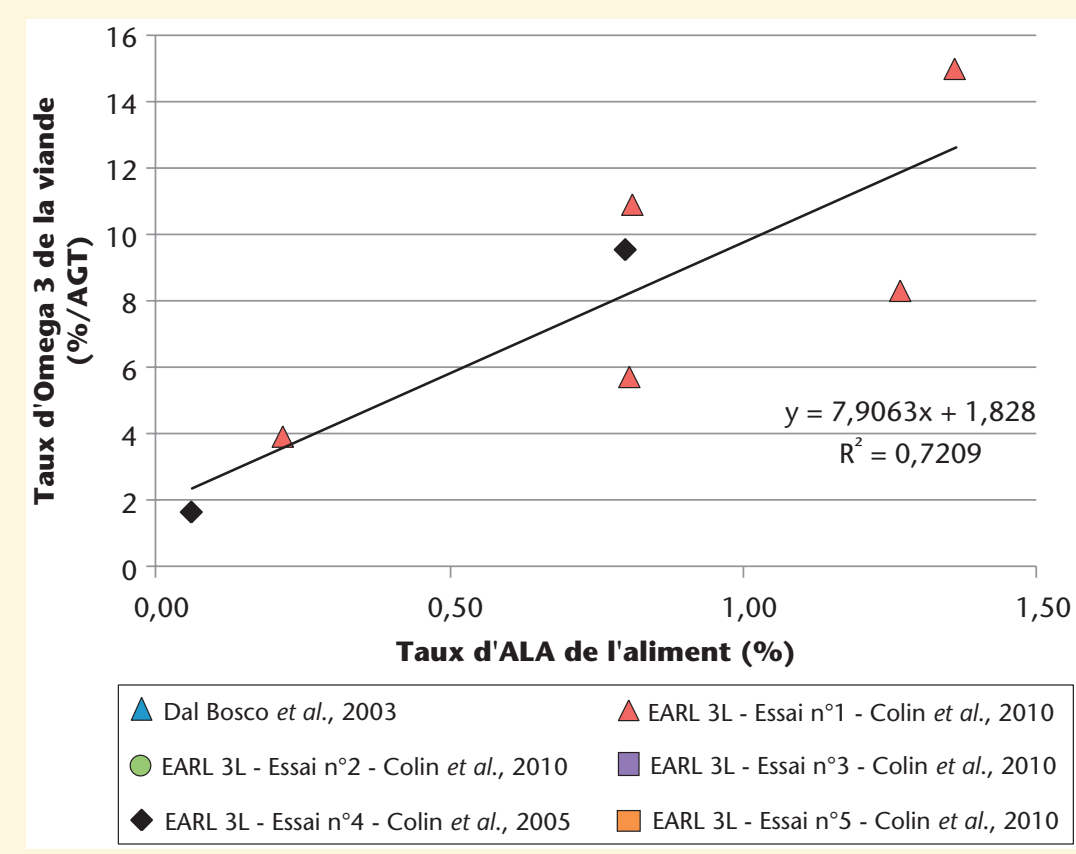

Figure 3. Relation entre la teneur en ALA de I'aliment et la teneur en ALA des graisses de la cuisse.

Un apport d'environ 0,65\% d'ALA dans I'aliment sous forme de graines de lin extrudées permet donc d'apporter en moyenne :

- pour le râble, 967 mg d'oméga-3 pour $100 \mathrm{~g}$ ou $459 \mathrm{mg}$ pour $100 \mathrm{kcal}$; - pour l'épaule, 1004 mg d'oméga-3 pour $100 \mathrm{~g}$ ou $483 \mathrm{mg}$ pour $100 \mathrm{kcal}$; - pour la cuisse, seulement $63 \mathrm{mg}$ d'oméga-3 pour $100 \mathrm{~g}$ ou $66 \mathrm{mg}$ pour $100 \mathrm{kcal}$ (tableau 4).

Ainsi le râble et l'épaule peuvent prétendre à l'allégation "riche en oméga-3 ».

\section{Conséquence sur les qualités hédoniques de la viande de lapin}

Les différents tests hédoniques réalisés lors de l'essai 4 et 5 n'ont montré aucune différence entre les 2 types de régimes (tableau 5). L'acceptabilité légèrement meilleure signalée par Dal Bosco et al. (2003) en faveur du lot enrichi en ALA (les autres paramètres restant inchangés) n'a pas été retrouvée dans les essais de l'EARL 3L. Les rares consommateurs ayant signalé l'existence d'un arrière goût (de 2 à
7 sur 60 selon les morceaux et les traitements) ont eu des opinions totalement divergentes quant aux goûts mentionnés (amer, sang, bouchon, chimique, fumé, poulet, porc, gibier) et les proportions étaient équivalentes pour les lapins des 2 origines. L'hypothèse du développement de saveurs désagréables suite à l'oxydation des acides gras insaturés paraît donc infirmée par ces 2 essais, en rapport probablement avec la présence dans la graine de lin des lignanes aux propriétés antioxydantes. II conviendra évidemment de vérifier cette affirmation par un dosage des composés d'oxydation primaire ou secondaire lors d'un essai ultérieur.

\section{Conclusion}

Cette synthèse démontre une relation directe entre l'apport d'acide linolénique de l'aliment et le taux d'oméga-3 de la viande de lapin et cela quelque soit le morceau. Cet enrichissement permet d'abaisser à la fois le taux d'acides gras saturés, le taux d'oméga 6 et ainsi le rapport oméga6/oméga-3. L'augmentation du taux d'ALA dans l'aliment permet ainsi d'obtenir des produits (le râble et l'épaule par exemple) à forte teneur en oméga-3 pour $100 \mathrm{~g}$ ou $100 \mathrm{kcal}$ de viande sans modification des caractéristiques hédoniques des produits. Ces essais ont également permis de vérifier que la très grande majorité de ces oméga-3 sont de I'ALA, les taux d'oméga-3 à longue chaîne restant faibles. De tels niveaux permettent d'alléguer sur la teneur en oméga- 3 de cette viande et notamment de revendiquer l'allégation "riche en oméga-3 ",

Tableau 3. Description des aliments;

\begin{tabular}{|c|c|c|c|c|c|c|c|}
\hline \multicolumn{2}{|c|}{ Type de lapins } & \multicolumn{3}{|c|}{ Lipides (\%) } & \multicolumn{3}{|c|}{ Protéines (\%) } \\
\hline & & Std & Lin & $\begin{array}{l}\text { Combes et Dal } \\
\text { Zotte, } 2005\end{array}$ & Std & Lin & $\begin{array}{l}\text { Combes et Da } \\
\text { Zotte, } 2005\end{array}$ \\
\hline \multirow{2}{*}{ Râbles } & Moy. & 11,9 & 13,6 & 11,4 & 20,2 & 19,6 & 19,7 \\
\hline & C.V. & n.d. & 40,6 & n.c. & n.d. & 4,6 & n.c. \\
\hline \multirow{2}{*}{ Épaule } & Moy. & 8,5 & 15,3 & 11,4 & 19,2 & 17,8 & 18,3 \\
\hline & C.V. & n.d. & 23,8 & n.c. & n.d. & 9,7 & n.c. \\
\hline \multirow{2}{*}{ Cuisse } & Moy. & 2 & 1,1 & 3,7 & 21,8 & 21,2 & 21,3 \\
\hline & C.V. & n.d. & 18,4 & 13 & n.d. & 0,8 & 4 \\
\hline
\end{tabular}


Tableau 4. Composition en lipides et protéines de la viande

\begin{tabular}{|c|c|c|c|c|c|c|c|c|c|c|c|c|}
\hline \multirow[b]{2}{*}{ Type de lapins } & \multicolumn{4}{|l|}{ Râble } & \multicolumn{4}{|c|}{ Épaule } & \multicolumn{4}{|c|}{ Cuisse } \\
\hline & Std & Lin & & & Std & Lin & & & Std & Lin & & \\
\hline Nombre d'analyses & 2 & 9 & & & 1 & 44 & & & 1 & 5 & & \\
\hline \multirow[t]{2}{*}{ Nombre de lapins } & 45 & 105 & & & 35 & 411 & & & 35 & 55 & & \\
\hline & Moy. & Moy. & E.T. & C.V. & Moy. & Moy. & E.T. & C.V. & Moy. & Moy. & E.T. & C.V. \\
\hline ALA (\%) dans I'aliment & 0,05 & 0,66 & 0,05 & 7,8 & 0,06 & 0,63 & 0,04 & 6,6 & 0,06 & 0,69 & 0,06 & 9,1 \\
\hline \multicolumn{13}{|l|}{ Composition générale } \\
\hline Humidité (\%) & 67,7 & 66,1 & 3,9 & 5,8 & 70,2 & 65,1 & 3,0 & 4,6 & 73,6 & 75,5 & $n c$ & $n c$ \\
\hline Lipides (\%) & 11,9 & 13,6 & 5,5 & 40,6 & 8,5 & 15,3 & 3,6 & 23,8 & 2,0 & 1,1 & 0,2 & 18,4 \\
\hline Protéines (\%) & 20,2 & 19,6 & 0,9 & 4,6 & 19,2 & 17,8 & 1,7 & 9,7 & 21,8 & 21,2 & 0,2 & 0,8 \\
\hline \multicolumn{13}{|l|}{ Composition des lipides } \\
\hline AGS (\%) & 36,0 & 31,4 & 2,9 & 9,1 & 37,8 & 33,6 & 3,2 & 9,6 & 36,8 & 34,6 & 2,2 & 6,3 \\
\hline AGMI (\%) & 32,5 & 37,0 & 7,1 & 19,2 & 30,9 & 38,4 & 6,8 & 17,7 & 34,2 & 33,3 & 3,7 & 11,2 \\
\hline AGPI (\%) & 31,0 & 31,4 & 5,9 & 18,8 & 30,8 & 27,2 & 5,1 & 18,6 & 28,2 & 31,9 & 3,8 & 11,8 \\
\hline Oméga-6 (\%) & 29,2 & 22,7 & 6,3 & 27,6 & 28,1 & 19,3 & 2,8 & 14,7 & 26,5 & 24,4 & 2,6 & 10,5 \\
\hline Oméga-3 (\%) & 1,5 & 8,5 & 1,2 & 14,3 & 1,7 & 7,8 & 3,1 & 39,7 & 1,6 & 7,2 & 1,4 & 19,1 \\
\hline Oméga-6/Oméga-3 & 19,4 & 2,7 & & & 16,5 & 2,5 & & & 16,6 & 3,4 & & \\
\hline \multicolumn{13}{|c|}{ Teneur dans $100 \mathrm{~g}$ ou $100 \mathrm{kcal}$ de viande } \\
\hline Oméga-3 (mg pour 100 g) & 155 & 967 & 414 & 42,8 & 121 & 1004 & 304 & 30,3 & 27 & 63 & 7 & 11,6 \\
\hline Oméga-3 (mg pour 100 kcal) & 83 & 459 & 122 & 26,7 & 79 & 483 & 153 & 31,6 & 26 & 66 & 8 & 11,4 \\
\hline
\end{tabular}

Tableau 5. Composition des lipides de la viande.

\begin{tabular}{|c|c|c|c|c|c|c|c|}
\hline & & $\begin{array}{l}\text { Râble } \\
\text { Standard }\end{array}$ & Lin & $\begin{array}{l}\text { Épaule } \\
\text { Standard }\end{array}$ & Lin & $\begin{array}{l}\text { Cuisse } \\
\text { Standard }\end{array}$ & Lin \\
\hline \multicolumn{2}{|c|}{ Nombre de consommateurs } & 60 & 60 & 60 & 60 & 30 & 30 \\
\hline \multirow{2}{*}{ Global } & Moyenne/7 & 4,10 & 4,30 & 4,60 & 4,55 & 4,60 & 4,20 \\
\hline & Écart-type & 1,79 & 1,50 & 1,53 & 1,55 & 1,30 & 1,60 \\
\hline \multirow{2}{*}{ Apparence } & Moyenne/7 & 4,25 & 4,70 & 4,50 & 4,45 & 4,80 & 4,70 \\
\hline & Écart-type & 1,63 & 1,57 & 1,68 & 1,54 & 1,40 & 1,50 \\
\hline \multirow[b]{2}{*}{ Odeur } & Moyenne/7 & 4,10 & 4,55 & 4,70 & 4,55 & 4,50 & 4,30 \\
\hline & Écart-type & 1,70 & 1,56 & 1,48 & 1,46 & 1,40 & 1,50 \\
\hline \multirow{2}{*}{ Goût } & Moyenne/7 & 4,10 & 4,35 & 4,55 & 4,50 & 4,40 & 4,00 \\
\hline & Écart-type & 1,74 & 1,63 & 1,57 & 1,64 & 1,40 & 1,50 \\
\hline \multirow{2}{*}{ Texture } & Moyenne/7 & 4,05 & 4,20 & 4,55 & 4,50 & 4,40 & 4,30 \\
\hline & Écart-type & 1,75 & 1,75 & 1,61 & 1,70 & 1,50 & 1,60 \\
\hline
\end{tabular}

très difficile à obtenir pour la plupart des produits carnés.

Remerciements. Les auteurs remercient la société Loeul-et-Piriot et en particulier Monsieur Philippe Rigaudy pour leur collaboration dans le prélèvement d'échantillons à l'abattoir.
Conflits d'intérêts : Les auteurs déclarent que leur société COPRI SARL développe une activité de vente de lin extrudé (Tradi-lin).

\section{RÉFÉRENCES}

Bernardini M, Castellini C, Dal Bosco A. Livello di omega 3 nella carne di coniglio in relazione al contenuto della dieta. In: Atti XII congresso Nazional ASPA, Pisa (Italia), 1997 ; 23-26.

Bernardini M, Castellini C, Dal Bosco A. Effect of dietary $n-3 / n-6$ ratio on fatty acid composition of liver, meat and perirenal fat in rabbits. Anim Sci 1999 ; 68 : 64754. 
Castellini C, Dal Bosco A. Effect of dietary herring meal on the omega-3 fatty acid content of rabbit meat. Proc. Symposium Food and Health : Role of animal products. Milano Ed Elsevier. 1997 ; 67-71.

Colin M, Raguenes N, Le Berre G, Charrier S, Prigent A-Y, Perrin G. Influence d'un enrichissement de I'aliment en Oméga 3 provenant de graines de lin extrudées (Tradi-

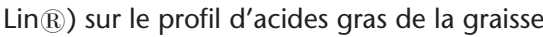
et sur les caractéristiques hédoniques de la viande de Lapin. In: 11e Journée de la Recherche Cunicole. Paris (France), 29-30 novembre 2005 : 163-6.

Colin M, Teillet B, Prigent AY. Qualités nutritionnelles de la viande de lapin recevant un aliment enrichi en acide alphalinolénique. In : $8^{e}$ Journées Francophones de Nutrition. Lille (France), 8-10 décembre 2010 : S97.
Colin M, Delarue J, Camino Callarisa A, et al. Efecto de la suplementacion del pienso con $\mathrm{DHA}$ vegetal en el crecimiento y la calidad de la carne del conejo. XXXVII Symposium de cunicultura. Barbastro (Espagne), 24-25 mai 2012 : 97-101.

Combes S, Dalle Zotte A. La viande de Lapin : valeur nutritionnelle et particularités physiologiques. 11ème Journée de la Recherche Cunicole. Paris (France), 29-30 novembre 2005 : 167-80.

Combes S, Cauquil L. Une alimentation riche en luzerne permet d'enrichir la viande des lapins en oméga 3. Viande et Produits Carnés 2006 ; 25, 1-5.

Dal Bosco A, Castellini C, Bianchi L, Mugnai C. Effect of dietary alpha-linolenic acid and vitamin $E$ on acidic composition, storage stability and sensory characteristics of rabbit meat. Meat Sci 2003 ; 66 : 406-13.
Dalle Zotte A. Propriétés spécifiques de la viande de Lapin. In : Jornadas internacionales de cunicultura. Vila Real (Portugal), 24-25 novembre 2000.

Gigaud V, Combes S. The effect of decreasing the omega 6/omega 3 ratio in feed on fatty acid content of rabbit meat to meet human dietary recommendations. In : $9^{\text {th }}$ World Rabbit Congress. Vérone (Italie), 10-13 juin 2008 : 1353-8.

Mourot J. Que peut-on attendre des pratiques d'élevage pour la viande de porcs et autres monogastriques ? OCL $2010 ; 17$ : 39-42.

Weill P, Chesneau G, Normand J, Mourot J, Colin M. Qualité lipidique des viandes. Effet du régime ou de l'espèce ? Quelques observations sur bovins, porcs, lapins et poulets. Nutr Clin Metab 2004 ; $18: 71$. 